1 London

Cite this as: BMJ 2021;375:n3133 http://dx.doi.org/10.1136/bmj.n3133 Published: 21 December 2021

\section{We need increased targeted measures now to slow the spread of omicron}

\author{
The government's current strategy of playing for time is a dangerous gamble, writes Sarah Hallett \\ Sarah Hallett BMA junior doctors committee co-chair and paediatric registrar?
}

“But omicron isn't as severe. The number of people in hospital or dying is nowhere near as high as we've seen before. Why all the fuss?"

These seem to be the current overriding sentiments in public discourse about why the huge surge in covid cases we are seeing isn't something to be too concerned about. Most healthcare staff, however, have been watching the exponential rise in covid cases and the spread of omicron with a sense of foreboding.

Firstly, it's unfortunately too early to draw any conclusions about severity. We simply don't yet know how unwell most people will be with omicron when compared to delta; Chris Whitty, the chief medical officer for England commented that while much is unknown about omicron, "all the things that we do know, are bad." Furthermore, the impact of a surge in cases takes a few weeks to be felt, both in terms of hospital admissions and mortality.

What we can say is that a small proportion of an incredibly large number is still a big number. If only a small percentage of the rising cases result in people having to be treated in hospital, then that will still increase the already immense pressure on the NHS, at a time when the system can least cope.

Most concerning, however, is what the case surge in the community means for our health and care workforce. Doctors, nurses and their colleagues do not exist in parallel to the rest of the population, and therefore we can assume that the more people who become ill with covid, the more NHS staff will likewise become ill, meaning they must stay home and cannot be at work to care for their patients.

This is already having an impact in London, where we've heard that around $10 \%$ of staff in one hospital are off with covid, and absences across the city are doubling in less than a week-with a further bleak assessment suggesting that one in three staff could be absent due to the virus by New Year. ${ }^{12}$

The BMA's own analysis, based on current case levels and trends, estimates that without further action to reduce the spread, we could have at best 30 ooo and at worst 130 ooo healthcare workers off sick because of covid by Christmas Day. ${ }^{3}$

The impact that this could have on patient care does not bear thinking about.

And that's not just about care for covid patients. We have a record almost six million people waiting for elective care in England-a number the government admits is likely to go up before it goes down. With fewer staff to tackle this backlog, it will grow at an even faster rate. For patients, hundreds of thousands of whom will have waited longer than a year to be seen, a further delay will have a devastating effect on their daily lives.

As in the wider health service, general practice faces sky-high demand and a historic workforce crisis, with practices already being asked to prioritise care so they can free-up time to take part in the booster campaign. Losing even more staff to sickness would therefore hit not just services available to patients in their surgeries, but also the very ability to deliver the vaccine programme.

Throughout the pandemic, we have seen doctors and colleagues redeployed to focus on managing surges in covid-19 cases, but the resources we have are finite-the most precious of which is our staff. Someone, somewhere will be missing out. And for junior doctors, it means missing out on valuable training and supervision that are so vital if we're to build a well rounded and sustainable medical workforce for the future.

Moreover, 20 months into the pandemic, staff are exhausted. Almost 8 in 10 doctors told our recent survey they were anxious about winter, and most worryingly, more than $60 \%$ said they were living with a mental health condition caused by work. Staff are working at their limits and once again forcing them into unsustainable working practices risks breaking them entirely.

Boris Johnson, the prime minister says that the Westminster government "won't hesitate" to act to protect the NHS. Yet, hesitation is exactly what it is doing.

The BMA is clear that we need increased targeted measures now to slow the spread of omicron and to protect staff, both with increased standards of personal protective equipment (PPE) for those on the front line, and reductions in social mixing and the suspension of mass events that threaten to accelerate transmission.

Of course, there needs to be better financial support for industries and individuals affected, but by taking action earlier, it would mean the need for tighter restrictions later on are less likely.

The government's current strategy of playing for time is a dangerous gamble, which is likely to be disastrous for the country, the NHS, and the care that patients probably won't be able to receive. The time to act is now.

Competing interests: none declared 


\section{OPINION}

1 BBC News. Omicron: London hospitals in dangerous situation, consultant says. December 2021. https://www.bbc.co.uk/news/uk-england-london-59682569

2 HSJ. Staff absence forecasts reveal "bleak picture" for coming days.

https://www.hsj.co.uk/workforce/staff-absence-forecasts-reveal-bleak-picture-for-comingdays/7031582.article

3 The BMA. BMA warns that without further measures, NHS could face almost 50000 staff off sick with Covid-19 by Christmas Day. https://www.bma.org.uk/bma-media-centre/bma-warnsthat-without-further-measures-nhs-could-face-almost-50-000-staff-off-sick-with-covid-19-bychristmas-day 